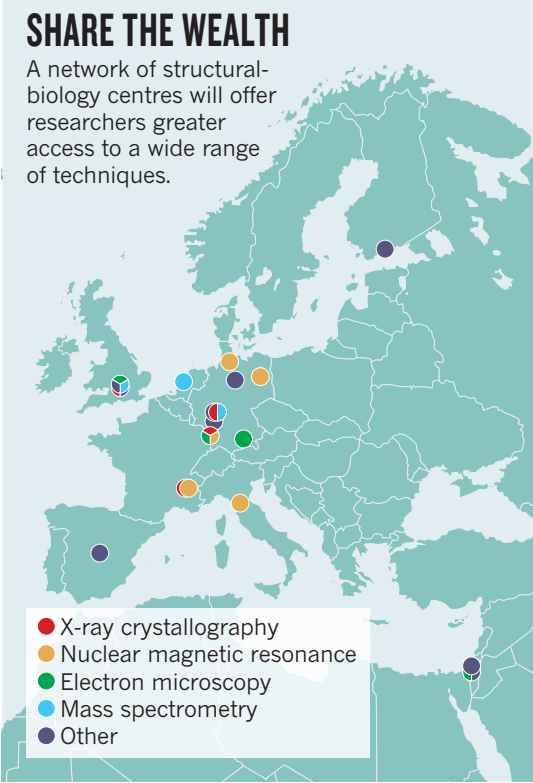

the subcellular compartments that the virus exploits. A crystal structure of a key molecular interaction might help to identify infection-blocking drugs.

"Only doing crystallography is not enough. Only doing electron microscopy is not enough. You often need all these technologies, and it's very hard to have them all in one house," says Albert Heck, scientific director of the Bijvoet Center for Biomolecular Research at Utrecht University in the Netherlands, which will offer mass spectrometry through Instruct.

Each of Instruct's facilities will cede up to $20 \%$ of their capacity to the programme (see 'Share the wealth'). Instruct will seek independent peer review of requests for equipment use and will approve access to various facilities, in some cases bypassing the allocation committees at individual centres.

The programme is initially using a subscription model, with eight nations, including Britain, France and Germany, agreeing to pay $€ 50,000$ per year to give their scientists access. Currently, the European Commission provides funding only for researchers to travel to these facilities. But Heck hopes that if Instruct is successful, Horizon 2020 will support both access and infrastructure for structural-biology centres, including equipment and staff, removing the need for subscriptions.

Aled Edwards, a structural biologist at the University of Toronto, Canada, is well used to juggling international resources as head of the Structural Genomics Consortium, which has solved more than 1,000 protein structures. He thinks Instruct makes sense. "It gives people access to technologies they don't have locally, it trains people," he says. "It's clearly setting itself up for Horizon 2020 funding. But they deserve it, if they can pull it off." -

REPRODUCTIVE BIOLOGY

\title{
Egg-making stem cells found in adult ovaries
}

\author{
Discovery could pave the way for new fertility treatments \\ and a longer reproductive life.
}

\section{BY KENDALL POWELL}

$\mathrm{I}$ t's time to rewrite the textbooks. For 60 years, everyone from high-school biology teachers to top fertility specialists has been operating under the assumption that women are born with all the eggs they will ever produce, with no way to replenish that supply. But the discovery of human egg-producing stem cells, harvested from the ovaries of six women aged 22 to 33, puts that dogma in doubt.

The work, published online in Nature Medicine $^{1}$ by Jonathan Tilly and colleagues at Massachusetts General Hospital in Boston, parallels the findings of a Shanghai-based group ${ }^{2}$ that isolated similar stem cells from mice in 2009. However, both this and Tilly's earlier work in mice $^{3}$ remained controversial, with many experts sceptical that such stem cells existed.

"This is unequivocal proof that not only was the mouse biology correct, but what we proposed eight years ago was also correct - that there was a human population of stem cells in young adult tissue," says Tilly.

To address the doubts, Tilly's team began by developing a more sensitive method for identifying and collecting mouse ovarian stem cells. Their method, based on a technique called fluorescence-activated cell sorting (FACS), attaches a fluorescently labelled antibody to a protein, Ddx4, that is present on the outer surface of the stem cells but not on the surface of the later-stage egg cells or oocytes. The FACS instrument lines up cells in single file and sorts them one by one, separating the labelled ones from the rest; it also gets rid of dead or damaged cells, such as oocytes, in which internal Ddx4 might become accessible to the antibody. This method is more selective than previous isolation methods, which did not get rid of such cells.

Once the team confirmed that it had isolated mouse ovarian stem cells by this method, it set its sights on reproductive-age human ovaries. Yasushi Takai, a former research fellow in Tilly's lab and now a reproductive biologist at Saitama Medical University in

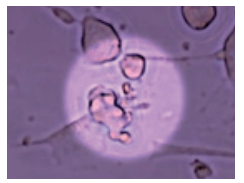

ONATURE.COM For a video explaining this research, see: go.nature.com/5ub5ma

Japan, supplied frozen whole ovaries removed from sex-reassignment patients, all young women of reproductive age. "It was 9 November when we did the first human FACS sort and I knew immediately that it had worked," says Tilly. "I cannot even put into words the excitement - and, to some degree, the relief - I felt."

The cells they pulled out, called oogonial stem cells (OSCs), spontaneously generated apparently normal immature oocytes when cultured in the lab. To look at the development of the putative human OSCs in a more natural environment, the team labelled the cells with green fluorescent protein to make them traceable, and injected them into fragments of adult human ovarian tissue, which were then transplanted under the skin of mice. After one to two weeks of growth, the OSCs had formed green-glowing cells that looked like

"I'veseenthese cells and how they behave. They're convincing and impressive." oocytes and that also expressed two of the genetic hallmarks of this cell type.

“There's no confirmation that we have baby-making eggs yet, but every other indication is that these cells are the real deal - bona fide oocyte precursor cells," says Tilly. The next step, to test whether the human OSC-derived oocytes can be fertilized and form an early embryo, will require special considerations - namely, private funding to support the work in the United States (federal funding cannot by law be used for any research that will result in the destruction of a human embryo, whatever the source of the embryo) or a licence from the UK Human Fertilisation and Embryology Authority to do the work with collaborators in the United Kingdom.

Evelyn Telfer, a reproductive biologist at the University of Edinburgh, UK, was once sceptical of the mouse work, but has become a believer. "I've visited [Tilly's] lab, seen these cells and how they behave. They're convincing and impressive," she says. Telfer, who studies the maturation of human eggs in vitro, will work with Tilly to try to grow the OSC-derived eggs to the point at which they are ready for fertilization.

She notes that there's still no evidence that the OSCs form new eggs naturally in the body. 
However, if they could be coaxed in a dish to make eggs that could successfully be used for in vitro fertilization (IVF), it would change the face of assisted reproduction.

"That's a huge 'if'," admits Tilly. But, he continues, it could mean an unlimited supply of eggs for women who have ovarian tissue that still hosts OSCs. This group could include cancer patients who have undergone sterilizing chemotherapy, women who have gone through premature menopause, or even those experiencing normal ageing. Tilly says that follow-up studies have confirmed that OSCs exist in the ovaries of women well into their 40s.

In addition, growing eggs from OSCs in the lab would allow scientists to screen for hormones or drugs that might reinvigorate these cells to keep producing eggs in the body and slow down women's biological clocks. "Even if you could gain an additional five years of ovarian function, that would cover most women affected by IVF," notes Tilly.

1. White, Y. A. R. et al. Nature Med. http://dx.doi. org/10.1038/nm.2669 (2012).

2. Zou, K. et al. Nature Cell Biol. 11, 631-636 (2009).

3. Johnson, J., Canning, J., Kaneko, T., Pru, J. K. \& Tilly, J. L. Nature 428, 145-150 (2004).

\section{TIMING TROUBLE}

Two possible sources of error may have affected the results of the OPERA experiment, which measures the arrival time of neutrinos speeding through Earth from CERN to Gran Sasso.
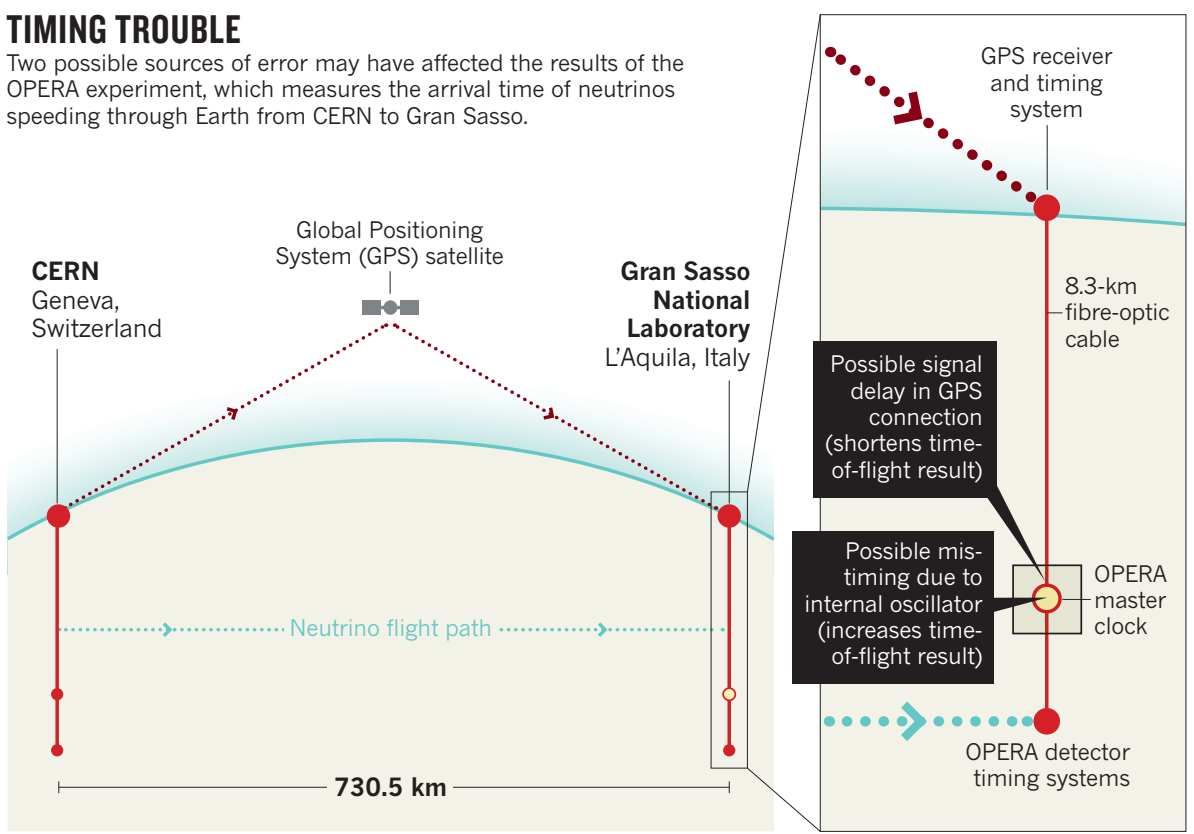

\section{PHYSICS}

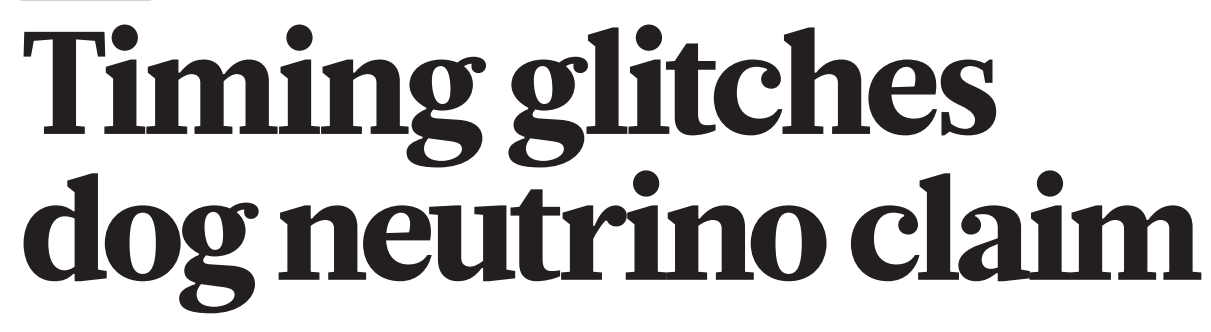

Team admits to possible errors in faster-than-light finding.

\section{BY EUGENIE SAMUEL REICH}

$\mathrm{I}$ $s$ it an epic blunder or a textbook demonstration of how science should work? To some physicists, the OPERA (Oscillation Project with Emulsion-tracking Apparatus) collaboration deserves credit for disclosing possible errors in its paradigm-challenging measurement of neutrinos travelling faster than light. "I think we did the right thing to continue to investigate," says Dario Autiero of the Institute of Nuclear Physics of Lyons in France, who presented the original results and notes that the collaboration had spent six months checking its result before its announcement last September.
To others, the revelation shows that the OPERA team went public too soon with its claim that neutrinos from CERN, the European particle-physics laboratory near Geneva in Switzerland, were flouting Albert Einstein's absolute limit on the speed of light as they travelled the 730 kilometres to the OPERA detector at the underground Gran Sasso National Laboratory near L'Aquila, Italy. "I find it embarrassing," says Luca Stanco of the National Institute of Nuclear Physics in Padova, Italy, an OPERA member who initially refused to sign a paper about the result. "Maybe we should have been more cautious and done more checks."

On 23 February, OPERA team members reported two possible sources of error in the experiment. The initial result suggested that the neutrinos were reaching the detector 60 nanoseconds faster than the speed of light would allow. Both potential errors would affect the neutrinos' arrival time, as measured by OPERA's master clock (see 'Timing trouble'). The first is a faulty connection at the point at which the light from a fibre-optic cable brings a synchronizing Global Positioning System (GPS) signal into the master clock. The fault could have delayed the GPS signal, causing the master clock to run slow and thus causing the neutrinos' travel time to appear shorter than it actually was.

"It's a subtle effect," says Autiero, and one that was evident only when the team examined many measurements of signals passing through the connection. Tests of the timing system turned up a second, opposing effect: an oscillator within the master clock that keeps time between the arrivals of synchronization signals was running fast. That would have made the neutrinos' travel time seem longer.

The collaboration says that it has not yet worked out the magnitude of these effects. Autiero says that because of the high profile of the result and the possibility of rumours and leaks, the collaboration wanted to disclose the potential errors promptly. The OPERA team plans to correct the faults and repeat the experiment after CERN's neutrino beam is switched on again in March, following a winter break.

Two independent checks of the measurement are also being considered. One, at Japan's Tokai to Kamioka (T2K) neutrino experiment, would still be valuable despite the doubt cast on the OPERA data, but may now prove harder to fund, says international co-spokesman Chang Kee Jung, a physicist at Stony Brook University in New York. But another, the Main Injector Neutrino Oscillation Search (MINOS) experiment, which fires neutrinos from Fermilab in Batavia, Illinois, to an underground detector in northern Minnesota, will proceed, at a cost of about US\$500,000. "It's never a bad idea to have multiple measurements," says MINOS co-spokesman Rob Plunkett.

Jorge Páramos, a physicist at the Higher Technical Institute in Lisbon, says that the admissions by OPERA point to an honest mistake, albeit one that should have been avoided. "The putative origin of the systematic error reflects the innards of the experiment - something that should have been checked exhaustively before any public announcement," he says. 